\title{
Theta Phase-dependent Modulation of Perception by Concurrent Transcranial Alternating Current Stimulation and Periodic Visual Stimulation
}

\author{
Elif Somer ${ }^{1}$, John Allen', Joseph Brooks ${ }^{1,2}$, \\ Vaughan Buttrill ${ }^{1}$, and Amir-Homayoun Javadi ${ }^{1,3,4}$
}

\begin{abstract}
Sensory perception can be modulated by the phase of neural oscillations, especially in the theta and alpha ranges. Oscillatory activity in the visual cortex can be entrained by transcranial alternating current stimulation (tACS) as well as periodic visual stimulation (i.e., flicker). Combined tACS and visual flicker stimulation modulates BOLD responses and concurrent 4-Hz auditory click trains, and tACS modulates auditory perception in a phase-dependent way. In this study, we investigated if phase synchrony between concurrent tACS and periodic visual stimulation (i.e., flicker) can modulate performance on a visual matching task. Participants completed a visual matching task on a flickering visual stimulus while receiving either in-phase $\left(0^{\circ}\right)$ or asynchronous $\left(180^{\circ}, 90^{\circ}\right.$, or $\left.270^{\circ}\right)$ tACS at alpha or theta frequency. Stimulation was applied over
\end{abstract}

\section{INTRODUCTION}

Previous research suggests that the state of oscillations in the brain may be a modulatory mechanism affecting sensory perception in both visual (e.g., Busch \& VanRullen, 2010; Busch, Dubois, \& VanRullen, 2009; Mathewson, Gratton, Fabiani, Beck, \& Ro, 2009) and auditory domains (e.g., Ng, Schroeder, \& Kayser, 2012; Lakatos et al., 2005; Rice \& Hagstrom, 1989). In particular, perceptual performance can vary according to the phase of neural oscillations at the time of stimulus presentation (e.g., Mathewson et al., 2009). Using brain stimulation techniques such as transcranial alternating current stimulation (tACS) to apply weak oscillatory electrical currents over the scalp (Thut, Schyns, \& Gross, 2011; Kanai, Chaieb, Antal, Walsh, \& Paulus, 2008) can modulate neural oscillations (Thut et al., 2011). Sensory stimulation, especially when periodic (i.e., flickering), can also affect neural oscillations. For instance, visual flicker stimulation can affect visual cognitive processes (e.g., Herbst, Javadi, van der Meer, \& Busch, 2013; Herrmann, 2001). Steady-state visual evoked potentials (SSVEPs) are oscillatory neural responses to rhythmic visual

\footnotetext{
${ }^{1}$ University of Kent, ${ }^{2}$ Keele University, ${ }^{3}$ University College
} London, ${ }^{4}$ Tehran University of Medical Sciences either occipital cortex or dorsolateral pFC. Visual performance was significantly better during theta frequency tACS over the visual cortex when it was in-phase $\left(0^{\circ}\right)$ with visual stimulus flicker, compared with antiphase $\left(180^{\circ}\right)$. This effect did not appear with alpha frequency flicker or with dorsolateral pFC stimulation. Furthermore, a control sham group showed no effect. There were no significant performance differences among the asynchronous $\left(180^{\circ}\right.$, $90^{\circ}$, and $270^{\circ}$ ) phase conditions. Extending previous studies on visual and auditory perception, our results support a crucial role of oscillatory phase in sensory perception and demonstrate a behaviorally relevant combination of visual flicker and tACS. The spatial and frequency specificity of our results have implications for research on the functional organization of perception.

stimuli observed in the EEG at the flicker frequency (and its harmonics) primarily over the posterior scalp (e.g., Spaak, de Lange, \& Jensen, 2014; Herbst et al., 2013; Brooks \& Palmer, 2011; Herrmann, 2001). Furthermore, both sensory and transcranial stimulation techniques were shown to be effective in phasic modulation of perception through entrainment of neural oscillations by EEG or magnetoencephalography recordings and behavioral measures (Jaegle \& Ro, 2014; Spaak et al., 2014; Henry \& Obleser, 2012; Neuling, Rach, Wagner, Wolters, \& Herrmann, 2012).

Chai, Sheng, Bandettini, and Gao (2018) combined tACS with periodic visual stimulation. They reported BOLD responses are modulated by tACS applied over Oz when it matched the visual flicker frequency or its second harmonic, mainly in areas activated by visual stimulation and targeted by the tACS current distribution. Furthermore, Riecke, Formisano, Herrmann, and Sack (2015) combined near-threshold 4-Hz click trains and tACS applied over the auditory cortices with varying difference angles $\left(30^{\circ}, 90^{\circ}, 150^{\circ}, 210^{\circ}, 270^{\circ}\right.$, and 330) between the auditory stimulus and tACS waveform. They found that perceptual detection accuracy oscillated at the tACS frequency, which is in line with other studies showing the phase of oscillations in the theta range is important for sensory perception (e.g., Tomassini, Ambrogioni, Medendorp, 
\& Maris, 2017; Ng et al., 2012; Busch \& VanRullen, 2010; Busch et al., 2009; Lakatos et al., 2005).

In this study, we used concurrent tACS applied over the early visual cortex (V1) or dorsolateral pFC (DLPFC) and visual stimulation in the theta $(4.1 \mathrm{~Hz})$ range to investigate if varying phase synchrony between them can affect perceptual performance in a visual matching task. We included DLPFC as a control location as it is associated with higher level cognitive processes such as short- and long-term memory (e.g., Crowley, Bendor, \& Javadi, 2019; Curtis \& D'Esposito, 2003; Javadi, Glen, Halkiopoulos, Schulz, \& Spiers, 2017; Javadi \& Walsh, 2012) Therefore, it would not be likely to affect lower level perceptual performance. We also investigated if alpha stimulation $(10 \mathrm{~Hz})$ would affect task performance as this is another frequency range that has been repeatedly linked to visual perception (e.g., Chai et al., 2018; Spaak et al., 2014; Busch et al., 2009; Mathewson et al., 2009; Kanai et al., 2008; Babiloni, Vecchio, Bultrini, Luca Romani, \& Rossini, 2005).

\section{METHODS}

\section{Participants}

The participants across all experiments were 105 University of Kent students (69 women, age range: 18-30 years; Experiment $1 n=30$, Experiment $2 n=30$, Experiment 3 $n=30$, Experiment $4 n=15$ ), who received course credits or $\$ 8$ as compensation for their time. All participants were informed about the brain stimulation but were unaware of the aim of the study, different frequencies, the existence of other stimulation location groups, and phase synchronization with the visual stimuli. Stimulation during the first practice block initiated any possible somatosensory feelings, giving the idea of stimulation to the participants. They were screened for past and present neurological conditions and a number of health conditions that would prevent them from safely receiving electrical brain stimulation. They did not report any condition that could prevent them from participating in the study. All participants gave written informed consent. The experimental procedures were approved by the School of Psychology Ethics Committee at the University of Kent.

\section{Stimuli and Apparatus}

The experiment was presented on a 23 -in. computer monitor $(60 \mathrm{~Hz}, 1920 \times 1080$ pixel resolution) using the experiment software PsychoPy (Peirce et al., 2019). The screen was situated at $60 \mathrm{~cm}$ viewing distance, and the background color was gray (red, green, blue: 160, $160,160)$. The visual display on each trial comprised four vertically oriented pseudorandom curvy lines (Figure 1) of $8.1^{\circ}$ in height and a maximum of $3.8^{\circ}$ in width. These were created using an algorithm from Brooks and Driver (2010). One target line appeared at the middle top of the display. Three curvy lines appeared in the bottom half of the display (Figure 1). One of these lines matched the target line, whereas the other two were modified versions of the target line. The position of the matching line was random on each trial. Stimuli remained on the screen until response. The color of the lines oscillated between magenta and the background gray (see the tACS and Visual Stimulation subsection). Following response, masks appeared at the site of each stimulus. These comprised magenta random shapes on a gray background (Figure 2).

\section{tACS and Visual Stimulation}

The electrical brain stimulation was delivered using a batteryoperated NeuroConn stimulation device (Germany). Nuprep skin preparation gel and saline solution were used to
Figure 1. Static example of visual stimulus with the target edge on top and three match options along the bottom. In this example, the rightmost stimulus on the bottom is the match to the target. The viewing distance was $60 \mathrm{~cm}$.

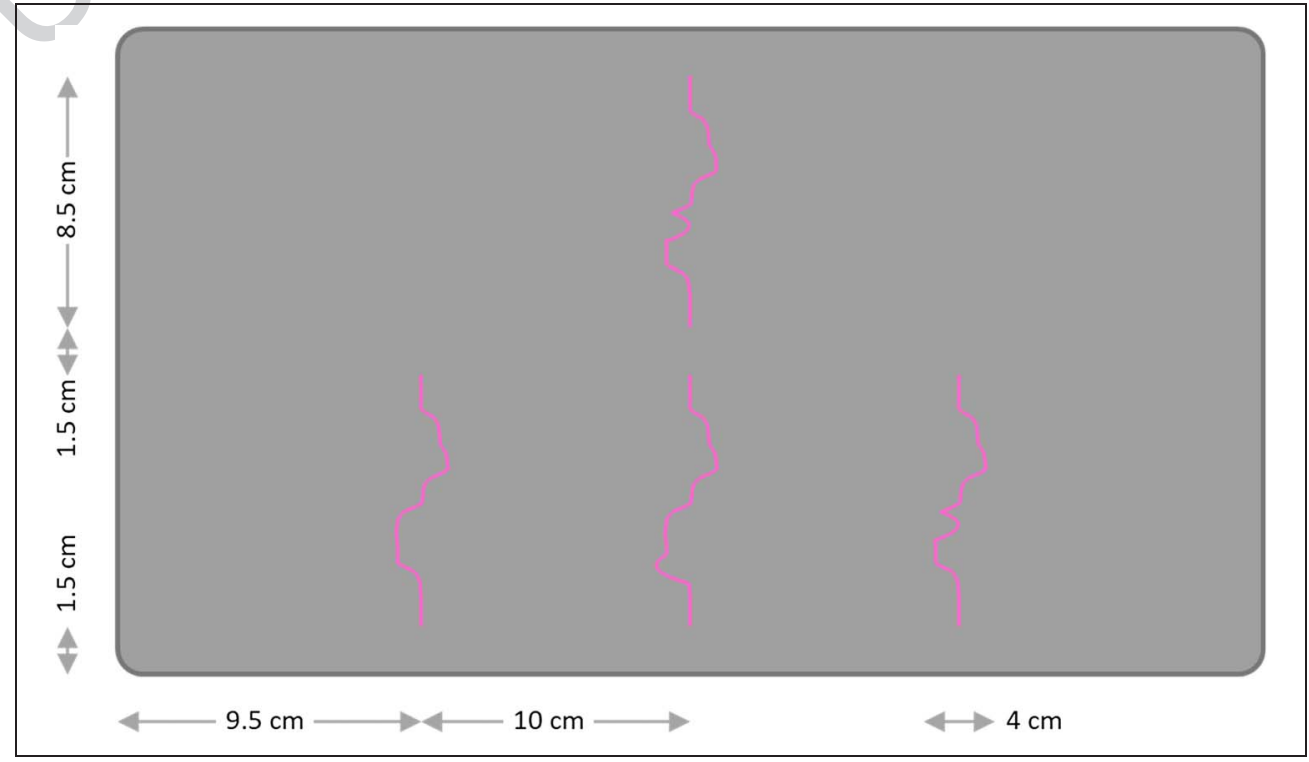




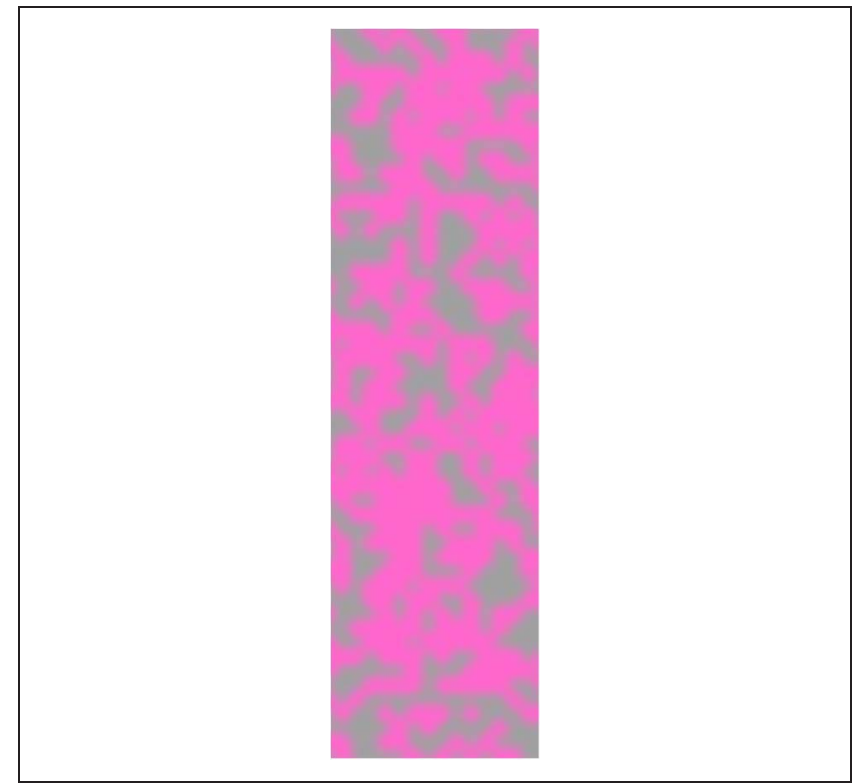

Figure 2. An example of the mask display.

improve conductivity. The area of stimulation was cleaned with skin preparation gel, and $5 \times 7 \mathrm{~cm}^{2}$ rubber electrodes placed inside sponges soaked in saline solution were attached to the participants' scalp and wrist using elastic bandages. The reference electrode was placed on the wrist as described by Accornero et al. (2014) and Fertonani and Miniussi (2017) to avoid possible interference with the stimulation and brain areas not of interest. The position of the electrodes attached to the scalp was defined by the 10-20 International EEG Electrode Placement System. For the participants in the occipital stimulation condition, the electrode was placed at position $\mathrm{Oz}$. For the participants in the DLPFC stimulation condition, the electrode was placed at position F3. The impedance was kept below $15 \mathrm{k} \Omega$. Alphafrequency stimulation was delivered at $10 \mathrm{~Hz}$, and thetafrequency stimulation was delivered at $4.1 \mathrm{~Hz}$ depending on the experiment. System testing indicated that $4.1 \mathrm{~Hz}$ was preferable to $4 \mathrm{~Hz}$ for the visual stimulation frequency in Experiment 1, as this ensured better characterization of the full range of luminosity (0-100\%) of the visual stimuli on the computer monitor used for these studies and it was felt that this slight deviation from convention would not make a material difference to other aspects of the study. The stimulation waveform always started at zero amps (i.e., zero phase). To create visual flicker, the color of the stimuli oscillated between magenta and the background gray at either 4.1 or $10 \mathrm{~Hz}$. Visual stimuli onset was at $0^{\circ}, 90^{\circ}, 180^{\circ}$, or $270^{\circ}$ phase relative to the tACS waveform. For instance, in the $0^{\circ}$-phase condition, the visual stimulus would start to ramp up toward full visibility at the $0^{\circ}$ position (zero amps ascending) of the tACS waveform. See the next section for details on how phase conditions were combined across experiments.

\section{Design and Procedure}

The first block of 130 trials were practice trials. No stimulation was applied in this block. Next, during the two stimulation blocks, a 4.1- or 10-Hz (depending on the experiment) sinusoidal current was applied at every trial. The stimulation was ramped up to $1.5 \mathrm{~mA}$ over four cycles at the beginning of each miniblock. Miniblocks lasted for either 10 trials or $30 \mathrm{sec}$, whichever was shorter depending on the response speed of the participant. Visual stimuli oscillated (starting as the background color) throughout the task at the same frequency as tACS in that experiment. The phase of the onset of the visual stimuli relative to tACS was randomly selected on each trial. The phases used varied across experiments (see below). Miniblocks were separated by $8 \mathrm{sec}$. There were a total of 130 trials in each of the two stimulation blocks.

On each trial, participants performed a visual matching task. The participants were instructed to indicate which of three curvy lines matched the target using the left, down, or right arrow keys on a keyboard. They were instructed to respond as accurately and as fast as possible because we intended to use a combined speed-accuracy measure, but no time limit was set for them to respond. Each trial started with a 650-msec fixation cross followed by the stimuli. The stimuli stayed on the screen until the participant responded. After the participant responded, masks (see Stimuli and Apparatus section) were presented for $100 \mathrm{msec}$, followed by the next trial. Intertrial interval varied as a result of $\mathrm{RT}$ in the trial.

In Experiment 1, we used a $2 \times 2$ mixed-factors design, with 30 participants (15 in each stimulation location) completing a visual matching task with a theta frequency flickering visual stimulus while receiving tACS, at the same frequency, over either the occipital cortex or DLPFC. The factors were stimulation phase $\left(0^{\circ}, 180^{\circ}\right.$; within subjects) and stimulation location (DLPFC or occipital; between subjects).

In Experiment 2, to investigate if the observed effect would vary with asynchronous phase delays other than the antiphase $\left(180^{\circ}\right)$, we tested 30 more participants with $90^{\circ}$ and $270^{\circ}$ phase difference between visual stimulation and tACS.

In Experiment 3, we repeated Experiment 1 with 30 new participants to investigate if the effect we found can be observed with stimulation in the alpha range $(10 \mathrm{~Hz})$. All other aspects of the study were kept the same.

In Experiment 4, we ran a sham group using in-phase or antiphase labels randomly assigned to trials and occipital or DLPFC electrode placements without actual stimulation with 15 participants.

\section{Statistical Analysis}

The participants' RTs and accuracy at every trial were recorded. The inverse efficiency score (IES; mean RT by the proportion of correct responses [PC]; Bruyer \& 


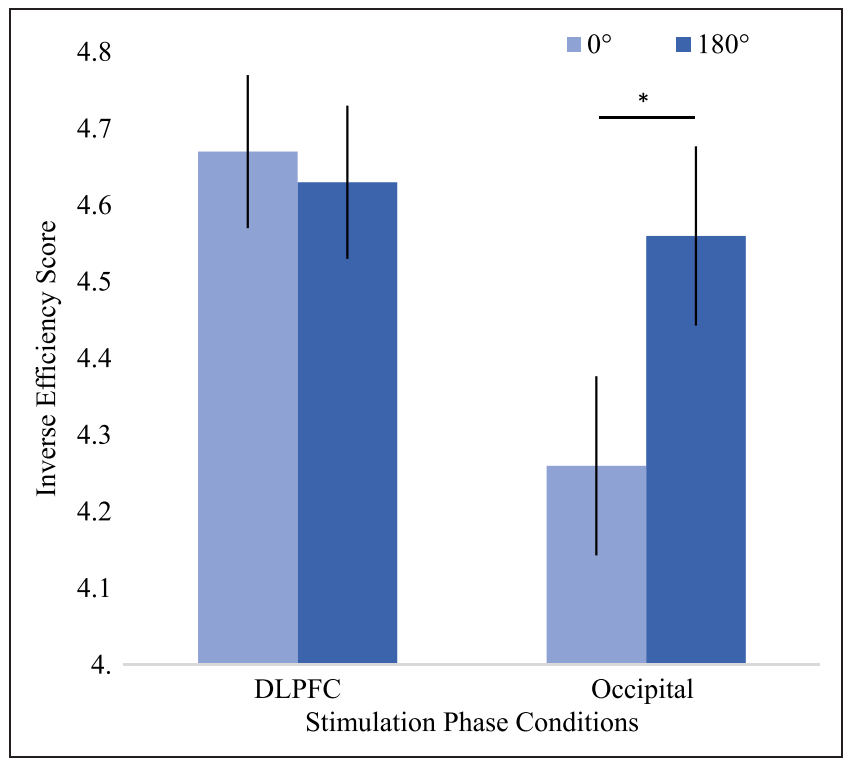

Figure 3. Estimated marginal means of IESs in stimulation phase conditions by stimulation location groups in Experiment 1. Error bars represent $1.96 S E$ adjusted for related means.

Brysbaert, 2011; Townsend \& Ashby, 1978) was calculated and used in the subsequent analyses. We used an alpha level of .05 for all statistical tests. The assumption of homogeneity of variance were met (Levene's tests, $p>.05$ ) for all of the statistical analyses unless stated otherwise. There were violations of the normality assumption (Shapiro-Wilk tests, $p<$ $.05)$ in some of the conditions. However, the group sizes were equal (or almost equal in Experiment 4), and no simple main effects tests were conducted for these groups.

\section{Phosphene and Somatosensation Control Study}

To investigate subjective perception of flicker, phosphenes, and somatosensation induced by the tACS, we ran a control study. Twelve participants (all women; mean age $=18.05$ years, $S D=0.15$ ) took part in this study. The design consisted of four interleaved blocks: two blocks of stimulation of the occipital area and two blocks of stimulation the left DLPFC. There was a 1-min break in between the blocks. Each block consisted of sixteen 20 -sec episodes of stimulation. Four combinations of stimulation protocols were used with different frequencies (alpha/theta) and duration (20 sec [active]/ $4 \mathrm{sec}[\mathrm{sham}])$ of stimulation. These conditions were pseudorandomized to avoid occurrence of one stimulation combination more than twice in a row. Each episode of stimulation was followed by a 20 -sec rest. Participants were instructed to look at a static screen containing a sample screen as shown in Figure 1. At the end of each episode, participants were asked to rate their perception of flicker, phosphenes, and somatosensation under both head and wrist electrodes on a 0-9 scale with 0 and 9 indicating "not at all" and "extremely strongly," respectively. Phosphenes were described to participants as flashes of light or distortions of the visual field (Schaeffner \& Welchman, 2017; Marg \& Rudiak, 1994). Flickers were described as changes in brightness. Somatosensation under the electrodes were described as scratches and burning sensations.

\section{RESULTS}

\section{Experiment 1: Modulation of Perception by Phase Synchrony}

The results of Experiment 1 showed that differences in phase synchrony between visual stimulation and tACS affected perceptual performance. A mixed design $2 \times 2$ ANOVA with stimulation phase $\left(0^{\circ}, 180^{\circ}\right)$ as a withinsubject factor, stimulation location (occipital, DLPFC) as a between-subject factor, and task performance (IES: see Methods section) as the dependent variable was conducted. There was a significant interaction between stimulation phase and location, $F(1,28)=4.72, p=.038, \eta_{\mathrm{p}}^{2}=$ .14 (see Figure 3 ), but no significant main effect of stimulation phase, $F(1,28)=2.72, p=.110, \eta_{\mathrm{p}}^{2}=.09$, or location, $F(1,28)=0.29, p=.596, \eta_{\mathrm{p}}^{2}=.01$. Pairwise comparisons with Bonferroni corrections revealed that performance was better in the $0^{\circ}$ compared with $180^{\circ}$ phase condition in the occipital group, $t(14)=2.70$, $p=.012, d=0.70$, but there was no significant difference for the DLPFC group, $t(14)=0.37, p=.714, d=$ 0.09 (see Table 1 for the descriptive statistics and estimated mean and standard error values). Levene's test of equality of error variances was significant, $p=.022$, for the $180^{\circ}$ phase condition. However, the main effect of the between-subject factor and pairwise comparisons between occipital and DLPFC groups for both $180^{\circ}, t(28)=$ $0.14, p=.889, d=0.04$, and $0^{\circ}, t(28)=0.98, p=$ $.334, d=0.25$, conditions were already not significant.

As we used a combined measure of RT and accuracy for our main analyses, we conducted additional analyses to investigate the source of the phase synchrony effect further. Our results indicate that the observed effect

Table 1. Descriptive Statistics (Mean $[S D]$ ) of the IES for Stimulation Phase Conditions according to Stimulation Location Groups

\begin{tabular}{lrrr}
\hline Experiment & Phase & Occipital & DLPFC \\
\hline Experiment 1 & $0^{\circ}$ & $4.26[1.33]$ & $4.67[0.93]$ \\
& $180^{\circ}$ & $4.56[1.64]$ & $4.63[0.92]$ \\
Experiment 2 & $90^{\circ}$ & $4.13[0.91]$ & $4.05[1.27]$ \\
& $270^{\circ}$ & $4.07[0.89]$ & $4.20[1.23]$ \\
Experiment 3 & $0^{\circ}$ & $4.41[1.60]$ & $4.33[1.15]$ \\
& $180^{\circ}$ & $4.45[1.68]$ & $4.16[1.09]$ \\
Experiment 4 & $0^{\circ}$ & $3.99[1.61]$ & $4.04[0.87]$ \\
& $180^{\circ}$ & $4.10[1.80]$ & $4.05[0.85]$ \\
\hline
\end{tabular}




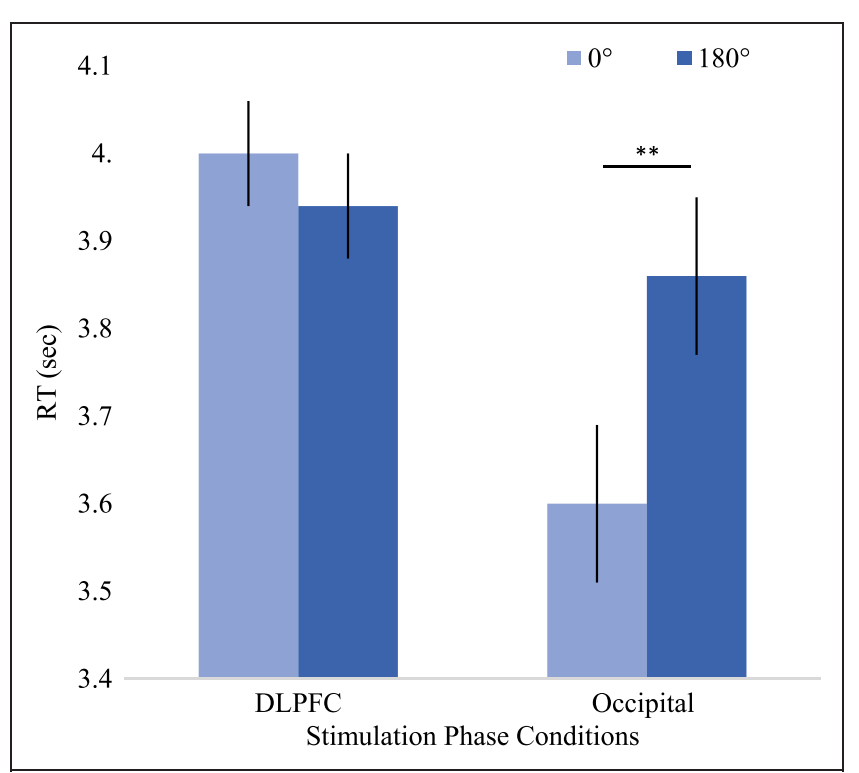

Figure 4. Estimated marginal means of RTs in stimulation phase conditions by stimulation location groups in Experiment 1. Error bars represent $1.96 S E$ adjusted for related means.

was mainly driven by RT rather than accuracy. A mixed design $2 \times 2$ ANOVA was repeated with stimulation phase $\left(0^{\circ}, 180^{\circ}\right)$ as a within-subject factor, stimulation location (occipital, DLPFC) as a between-subject factor, and task performance (RT) as the dependent variable. There was a significant interaction effect between stimulation phase and location, $F(1,28)=7.82, p=.009, \eta_{p}^{2}=.22$ (see Figure 4), but no significant main effect of stimulation phase, $F(1,28)=2.92, p=.099, \eta_{\mathrm{p}}^{2}=.09$, or location, $F(1,28)=0.26, p=.616, \eta_{\mathrm{p}}^{2}=.01$. Performance was worse in the $180^{\circ}$ compared with $0^{\circ}$ phase condition in the occipital group, $t(14)=3.20, p=.004, d=0.83$, and there was again no significant difference for the DLPFC group, $t(14)=0.77, p=.448, d=0.20$, as shown by pairwise comparisons with Bonferroni corrections (see Table 2 for the descriptive statistics and estimated mean and standard error values). Levene's test of equality of error variances was significant, $p=.014$, for the $180^{\circ}$ phase condition. However, the main effect of the between-subject factor and pairwise comparisons between occipital and DLPFC groups for both $180^{\circ}, t(14)=0.16, p=.873, d=0.04$, and

Table 2. Descriptive Statistics (Mean $[S D]$ ) of the RTs and PC for Stimulation Phase Conditions according to Stimulation Location Groups in Experiment 1

\begin{tabular}{lrcc}
\hline Location & Phase & $R T(\mathrm{sec})$ & $P C$ \\
\hline Occipital & $0^{\circ}$ & $3.60[1.40]$ & $0.83[0.15]$ \\
& $180^{\circ}$ & $3.86[1.67]$ & $0.82[0.14]$ \\
DLPFC & $0^{\circ}$ & $4.01[1.11]$ & $0.85[0.10]$ \\
& $180^{\circ}$ & $3.94[1.04]$ & $0.85[0.12]$ \\
\hline
\end{tabular}

$0^{\circ}, t(14)=0.88, p=.386, d=0.23$, conditions were already not significant.

A mixed design $2 \times 2$ ANOVA with stimulation phase $\left(0^{\circ}\right.$, $180^{\circ}$ ) as a within-subject factor, stimulation location (Occipital, DLPFC) as a between-subject factor, and task performance (PC) as the dependent variable revealed no significant interaction effect between stimulation phase and location, $F(1,28)=0.09, p=.773, \eta_{\mathrm{p}}^{2}<.01$, and no significant main effect of stimulation phase, $F(1,28)=0.05$, $p=.824, \eta_{\mathrm{p}}^{2}<.01$, or location, $F(1,28)=0.28, p=.603$, $\eta_{\mathrm{p}}^{2}=.01$ (see Table 2 for the descriptive statistics and estimated mean and standard error values).

\section{Experiment 2: No Significant Difference between Asynchronous Phase Conditions}

In Experiment 2, a mixed design $2 \times 2$ ANOVA with stimulation phase $\left(90^{\circ}, 270^{\circ}\right)$ as a within-subject factor, stimulation location (occipital, DLPFC) as a between-subject factor, and task performance (IES) as the dependent variable revealed no significant interaction effect between stimulation phase and location, $F(1,28)=2.92, p=$ $.098, \eta_{\mathrm{p}}^{2}=.10$, and no significant main effect of stimulation phase, $F(1,28)=0.70, p=.410, \eta_{\mathrm{p}}^{2}=.02$, or location, $F(1,28)=0.01, p=.946, \eta_{\mathrm{p}}^{2}=.00$ (see Table 1 for the descriptive statistics and estimated mean and standard error values; see Figure 5). Four independent-samples $t$ tests between the asynchronous phase conditions from Experiments 1 and 2 for the occipital and DLPFC groups were conducted. As Levene's tests indicated the assumption of equality of variances was violated, the degrees of freedom for the occipital group were adjusted. There were no significant differences between phases $180^{\circ}$ and $90^{\circ}$, or $270^{\circ}$ in the occipital group. There were also no significant

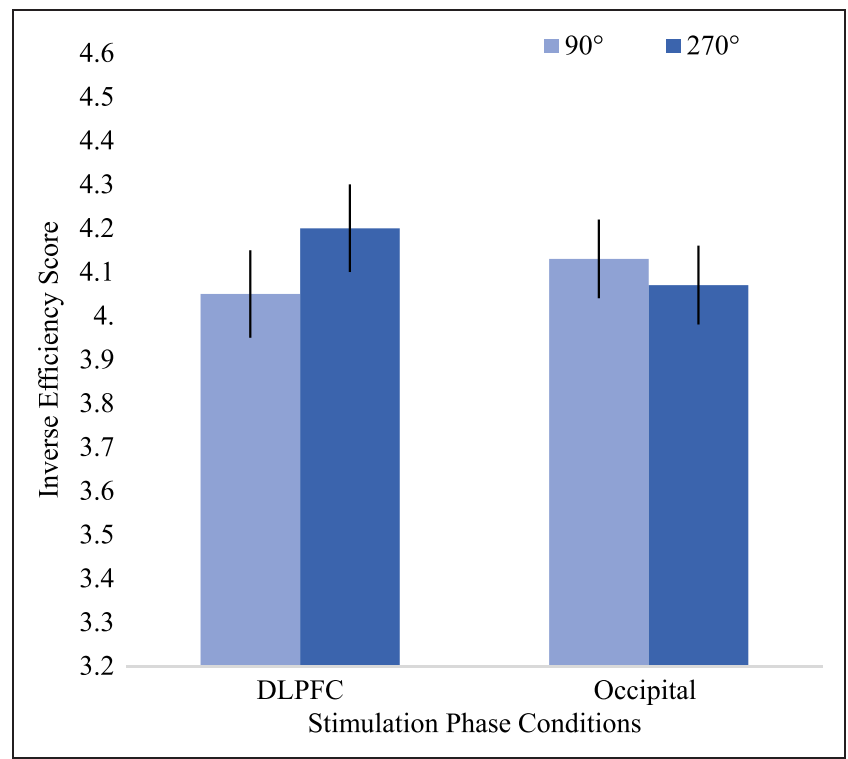

Figure 5. Estimated marginal means of IESs in stimulation phase conditions by stimulation location groups in Experiment 2. Error bars represent $1.96 S E$ adjusted for related means. 
Table 3. Summary of the Independent-samples $t$ Tests Comparing Phase Asynchronous Conditions from Experiments 1 and 2

\begin{tabular}{lcccc}
\hline Stimulation Location & Stimulation Phase & $t$ & $p$ & Cohen's d \\
\hline Occipital & $180^{\circ}-90^{\circ}$ & $t(21.80)=0.90$ & .378 & 0.33 \\
& $180^{\circ}-270^{\circ}$ & $t(21.61)=2.69$ & .323 & .37 \\
DLPFC & $180^{\circ}-90^{\circ}$ & $t(28)=1.43$ & .164 & .290 \\
& $180^{\circ}-270^{\circ}$ & $t(28)=1.08$ & 0.39 \\
\hline
\end{tabular}

differences between phases $180^{\circ}$ and $90^{\circ}$, or $270^{\circ}$ in the DLPFC group (see Table 1 for mean and standard deviation values and Table 3 for a summary of the independent samples $t$ tests).

\section{Experiment 3: Frequency Specificity of Effects}

In Experiment 3, there was no significant interaction effect between stimulation phase and location, $F(1,28)=$ $2.70, p=.111, \eta_{\mathrm{p}}^{2}=.09$, and no significant main effect of stimulation phase, $F(1,28)=1.07, p=.310, \eta_{\mathrm{p}}^{2}=.04$, or location, $F(1,28)=0.13, p=.720, \eta_{\mathrm{p}}^{2}=.01$, in a mixed design $2 \times 2$ ANOVA with stimulation phase $\left(0^{\circ}, 180^{\circ}\right)$ as a within-subject factor, stimulation location (occipital, DLPFC) as a between-subject factor, and task performance (IES) as the dependent variable (see Table 1 for the descriptive statistics and estimated mean and standard error values; see Figure 6).

\section{Experiment 4: No Significant Effect of Sham-tACS}

In Experiment 4, there was no significant interaction, $F(1,13)=$ $0.34, p=.571, \eta_{\mathrm{p}}^{2}=.03$, or main effects of assigned phase, $F(1,13)=0.41, p=.531, \eta_{\mathrm{p}}^{2}=.03$, or location, $F(1,13)=$

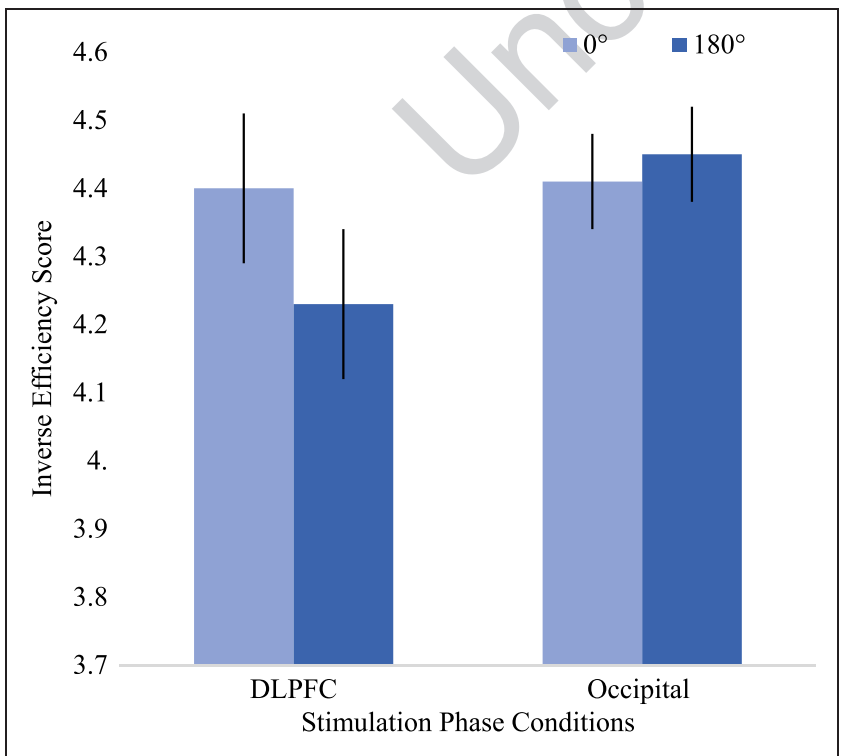

Figure 6. Estimated marginal means of IESs in stimulation phase conditions by stimulation location groups in Experiment 3. Error bars represent $1.96 S E$ adjusted for related means.
0.00, $p=.999, \eta_{\mathrm{p}}^{2}<.01$, in a mixed design $2 \times 2$ ANOVA with assigned phase $\left(0^{\circ}, 180^{\circ}\right)$ as a within-subject factor, electrode location (occipital, DLPFC) as a between-subject factor, and task performance (IES) as the dependent variable (see Table 1 for the descriptive statistics and estimated mean and standard error values; see Figure 7). Two independentsamples $t$ tests between $\mathrm{PC}$ in the $0^{\circ}$ phase condition from the sham $(M=0.82, S D=0.10)$, occipital theta $(M=0.82, S D=$ $0.15), t(28)=0.18, p=.862, d=0.07$, and DLPFC theta $(M=$ $0.85, S D=0.10)$ groups, $t(28)=0.82, p=.420, d=0.29$, showed no significant differences. Furthermore, there were also no significant differences between PCs in the $180^{\circ}$ phase condition from the sham $(M=0.82, S D=0.08)$, occipital theta $(M=0.82, S D=0.14), t(28)=0.07, p=.948$, $d=0.02$, and DLPFC theta $(M=0.85, S D=0.12)$ groups, $t(28)=0.63, p=.536, d=0.22$.

\section{Cross-experiment Comparisons}

A mixed design $2 \times 2 \times 3$ ANOVA was conducted with stimulation phase $\left(0^{\circ}, 180^{\circ}\right)$ as a within-subject factor, stimulation location (occipital, DLPFC) as first betweensubject factor, experiment groups (theta, alpha, control)

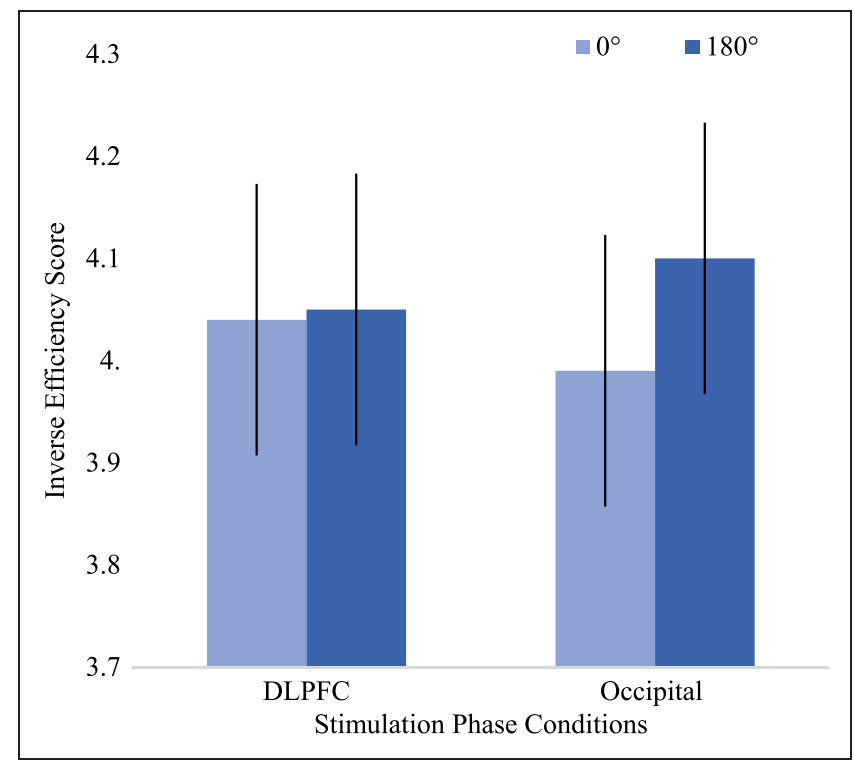

Figure 7. Estimated marginal means of IESs in stimulation phase conditions by stimulation location groups in Experiment 4 . Error bars represent $1.96 S E$ adjusted for related means. 
Table 4. Summary of the Results of the Control Study Investigating Subjective Perception of Phosphene, Flicker, and Somatosensation

\begin{tabular}{|c|c|c|c|c|c|}
\hline \multirow[b]{2}{*}{ Brain Area } & \multirow[b]{2}{*}{ Perception } & \multicolumn{2}{|c|}{ Theta } & \multicolumn{2}{|c|}{ Alpha } \\
\hline & & Sham & Active & Sham & Active \\
\hline \multirow[t]{4}{*}{ F3 } & Flicker & $0.59[0.33]$ & $0.52[0.34]$ & 2.79 [2.61] & $5.84[2.17]$ \\
\hline & Phosphene & $0.51[0.31]$ & $0.94[1.12]$ & 2.88 [2.69] & $5.26[2.69]$ \\
\hline & Head & $2.48[1.52]$ & $4.82[2.14]$ & 3.08 [1.14] & $4.97[1.14]$ \\
\hline & Wrist & $1.48[1.60]$ & $2.07[2.46]$ & 2.17 [2.61] & 2.05 [1.78] \\
\hline \multirow[t]{4}{*}{$\mathrm{Oz}$} & Flicker & $0.40[0.33]$ & $0.59[0.60]$ & $0.67[0.59]$ & $0.71[0.27]$ \\
\hline & Phosphene & $0.40[0.33]$ & $0.68[0.58]$ & $0.75[0.82]$ & $1.29[1.11]$ \\
\hline & Head & 2.72 [2.59] & $3.17[1.82]$ & $3.29[2.53]$ & $3.66[2.91]$ \\
\hline & Wrist & 2.14 [2.27] & 3.08 [3.08] & 2.13 [2.82] & 2.07 [2.53] \\
\hline
\end{tabular}

Head and wrist indicate somatosensation under the head and wrist electrodes.

as second between-subject factor, and task performance (mean RT) as the dependent variable. There was a significant three-way interaction effect between stimulation phase, location, and experiment groups, $F(1,70)=5.19$, $p=.026, \eta_{\mathrm{p}}^{2}=.07$. When we break this interaction down and look at the Phase $\times$ Location interaction, for theta frequency group, there was a clear Phase $\times$ Location interaction (see Figure 4), $F(1,28)=5.19, p=.009, \eta_{\mathrm{p}}^{2}=.22$. In contrast, there were no significant Phase $\times$ Location interaction for alpha frequency and control groups: $F(1,28)=$ $0.42, p=.524, \eta_{\mathrm{p}}^{2}=.02$ and $F(1,13)=0.35, p=.571, \eta_{\mathrm{p}}^{2}=$ .06 , respectively.

The same analysis conducted with IES as dependent variable did not show a significant three-way interaction, $F(1,70)=0.42, p=.524, \eta_{p}^{2}=.02$, although the same pattern was obtained for Phase $\times$ Location interaction as in the RT mean: $F(1,28)=4.72, p=.038, \eta_{\mathrm{p}}^{2}=.14$ for theta frequency; $F(1,28)=1.07, p=.310, \eta_{\mathrm{p}}^{2}=.04$ for alpha frequency; and $F(1,13)=0.34, p=.571, \eta_{\mathrm{p}}^{2}=.03$ for control group.

\section{Phosphene and Somatosensation Control Study}

Table 4 summarizes the subjective perception of the four measures of flicker, phosphene, and somatosensation underneath the head and wrist electrodes. To investigate whether the performance enhancement of occipital theta tACS could have been mediated by tACS-induced phosphenes or other artifacts, we compared sham- and active-tACS conditions for the two locations (F3/Oz) and frequency (alpha/theta) using paired-sample $t$ tests (see Table 5). The results indicate a pronounced perception

Table 5. Results of the Paired-sample $t$ Tests, Comparing Sham- and Active-tACS for the Four Measurements and Eight Conditions of Stimulation Location (F3/Oz), Frequency (Alpha/Theta), and Condition (Active/Sham) for the Control Study

\begin{tabular}{|c|c|c|c|c|c|c|c|}
\hline \multirow[b]{2}{*}{ Brain Area } & \multirow[b]{2}{*}{ Perception } & \multicolumn{3}{|c|}{ Theta } & \multicolumn{3}{|c|}{ Alpha } \\
\hline & & $t(11)$ & $p$ & $d$ & $t(11)$ & $p$ & $d$ \\
\hline \multirow[t]{4}{*}{ F3 } & Flicker & 0.54 & 0.599 & 0.16 & 2.53 & $0.028 *$ & 0.73 \\
\hline & Phosphene & 1.25 & 0.235 & 0.36 & 2.41 & $0.035^{*}$ & 0.70 \\
\hline & Head & 2.83 & $0.016^{*}$ & 0.82 & 4.80 & $0.001 * *$ & 1.39 \\
\hline & Wrist & 0.68 & 0.511 & 0.20 & 0.10 & 0.917 & 0.03 \\
\hline \multirow[t]{4}{*}{$\mathrm{Oz}$} & Flicker & 0.86 & 0.408 & 0.25 & 0.22 & 0.828 & 0.06 \\
\hline & Phosphene & 1.22 & 0.248 & 0.35 & 1.30 & 0.220 & 0.38 \\
\hline & Head & 0.42 & 0.682 & 0.12 & 0.29 & 0.778 & 0.08 \\
\hline & Wrist & 0.78 & 0.447 & 0.23 & 0.04 & 0.964 & 0.01 \\
\hline
\end{tabular}

$d$ represent Cohen's $d$ effect size.

$* p<.05$.

$* * p<.01$. 
of flicker and phosphenes only when alpha-tACS was applied to the left DLPFC. Somatosensation underneath the head electrode over the left DLPFC was also different between sham- and active-tACS in theta condition. The fact that these artifacts are only significantly greater with stimulation at the left DLPFC site, but not at the occipital stimulation site at which we observed our phase-dependent tACS effect, suggests that they cannot explain our effect.

\section{DISCUSSION}

We investigated the effects of varying phase synchrony between concurrent theta $(4.1 \mathrm{~Hz})$ or alpha $(10 \mathrm{~Hz})$ tACS and visual flicker applied over the occipital cortex or DLPFC. Our findings showed that phase synchrony between theta visual stimulation and tACS applied over the visual cortex modulates perceptual performance. Performance was significantly better in the in-phase $\left(0^{\circ}\right)$ stimulation condition compared with antiphase $\left(180^{\circ}\right)$ stimulation condition only in the theta occipital group. Cross-experiment comparisons showed a significant Phase $\times$ Location $\times$ Experiment interaction for RT but not for IES. This would be in line with our results being mainly driven by RT rather than accuracy. Participants from a control study reported noticeable phosphenes, but only during the DLPFC-alpha stimulation where there were no significant differences in performance in our main study. Our results are largely in line with Riecke et al. (2015), who showed phase synchrony between 4-Hz click trains and tACS-modulated auditory perception. However, they found performance was better for the stimuli presented during the positive half-wave (phases $30^{\circ}$ and $150^{\circ}$ ) of $4-\mathrm{Hz}$ tACS than the negative half-wave (phases $210^{\circ}-330^{\circ}$ ) while we found no difference between asynchronous phase conditions. Clouter, Shapiro, and Hanslmayr (2017), who showed better performance with phase synchrony between oscillating theta auditory and visual stimuli, also reported no difference between varying asynchronous phase offsets. However, their outcome measure was episodic memory. Our findings are unlikely to be due to a general tACS effect on motor performance as the phase of tACS relative to the visual stimulus would not be expected to affect performance in such case. Furthermore, electrophysiological evidence for the efficiency of tACS applied over the occipital cortex in phase-specific modulation of SSVEPs has recently been reported, although in the alpha-frequency range (Fiene et al., 2019). More broadly, our findings support the literature suggesting sensory perception is modulated by the phase of oscillations in the sensory cortices (e.g., Riecke et al., 2015; Neuling et al., 2012; Ng et al., 2012; Busch \& VanRullen, 2010; Busch et al., 2009; Mathewson et al., 2009; Lakatos et al., 2005; Rice \& Hagstrom, 1989) and the importance of theta range oscillations for perception (e.g., Tomassini et al., 2017; Riecke et al., 2015; Ng et al., 2012; Busch \& VanRullen, 2010; Busch et al., 2009; Lakatos et al., 2005).
Interestingly, the effect that we observed during theta stimulation was not present for alpha stimulation. Both theta (e.g., Spyropoulos, Bosman, \& Fries, 2018; Landau, Schreyer, Van Pelt, \& Fries, 2015; Demiralp et al., 2007; Lakatos et al., 2005) and alpha (e.g., Herring, Esterer, Marshall, Jensen, \& Bergmann, 2019; Spaak, Bonnefond, Maier, Leopold, \& Jensen, 2012) oscillations have been associated with the modulation of visual processing through modulating gamma oscillations. However, alpha oscillations were associated with selective attention on a single stimulus (e.g., Thut, Nietzel, Brandt, \& Pascual-Leone, 2006) and suppressing information from unattended regions (e.g., Händel, Haarmeier, \& Jensen, 2011). whereas distributed attention between two or more stimuli is facilitated by theta rhythmicity (e.g., Kienitz et al., 2018; Spyropoulos et al., 2018; Landau et al., 2015; Fiebelkorn, Saalmann, \& Kastner, 2013; Landau \& Fries, 2012). Our task required comparing at least two stimuli simultaneously while most studies focusing on alpha oscillations included perception of a single, often near-threshold stimulus at a time (e.g., Spaak et al., 2014; Busch et al., 2009; Mathewson et al., 2009; Babiloni et al., 2005; Rice \& Hagstrom, 1989). The need to investigate possible differences in the functions of alpha- and theta-frequency bands for the perceptual process depending on the task or stimuli has been discussed (Hanslmayr, Volberg, Wimber, Dalal, \& Greenlee, 2013). Our results might indicate different outcomes of the two frequency bands on perception. Future research can address if, with a single near-threshold stimuli detection task, the effect would be observed in the alpha range, although this could present challenges to delivering effective visual stimulation. Furthermore, our effect mainly stemmed from RT rather than accuracy. Distinct effects of attentional cueing characteristics on RT and accuracy have been shown, and they may represent different underlying processes (e.g., van Ede, de Lange, \& Maris, 2012; Prinzmetal, McCool, \& Park, 2005). However, further research would be needed to determine if our task characteristics interact with these two measures differently. Furthermore, research on cross-frequency phase coupling is being conducted (e.g., Scheffer-Teixeira \& Tort, 2016; Zheng, Bieri, Hsiao, \& Colgin, 2016; Xu, Liu, Li, \& Zhang, 2015; Belluscio, Mizuseki, Schmidt, Kempter, \& Buzsáki, 2012; Darvas, Miller, Rao, \& Ojemann, 2009; Isler, Grieve, Czernochowski, Stark, \& Friedman, 2008). It is possible that phase coupling between theta and alpha stimulation and a consequent behavioral effect could be achieved. We did not investigate this possibility as we have kept the visual flicker and electrical stimulation frequencies the same in all conditions to focus on phase-dependent effects. Researching this in the future can help clarify if coupling tACS in other frequencies with theta modulated stimuli or theta tACS with stimuli modulated in other frequencies can modulate visual perception.

As well as modulating visual attention and perception within the visual cortex by facilitating bidirectional communication and influence between lower and higher 
levels (Spyropoulos et al., 2018; Hanslmayr et al., 2013), neural oscillatory activity is a potential mechanism for dynamic organization and communication of cognitive processes of all levels across modalities and distant brain areas (Buzsáki \& Draguhn, 2004; Başar, Başar-Eroğlu, Karakaş, \& Schürmann, 2000, 2001; Engel, Fries, \& Singer, 2001), particularly in the theta range (Spyropoulos et al., 2018; Hanslmayr et al., 2013; Demiralp et al., 2007; Başar et al., 2000; Başar et al., 2001). It remains an open research question how the demonstrated effect would be reflected in later cognitive processes such as memory and what the effects of varying phase synchrony between sensory stimulation and tACS applied over parietal or frontal regions be on memory. This presents an opportunity for investigating the functional and anatomical structure of perceptual and memory processes and the relationship between them. Future research can also employ simultaneous tACS, visual stimulation, and magnetoencephalographic recording using a recently demonstrated novel approach (Ruhnau, Keitel, Lithari, Weisz, \& Neuling, 2016). Although SSVEPs are well documented (e.g., Spaak et al., 2014; Herbst et al., 2013; Herrmann, 2001) and there is emerging evidence of entrainment through tACS (e.g., Helfrich et al., 2014; Kanai et al., 2008), electrophysiological evidence would strengthen and further clarify our findings as discussed by Thut et al. (2011).

Also, one important possibility that should be considered is that the differences we observed between inphase and antiphase stimulation could be caused by peripheral effects of tACS such as phosphenes and not be of cortical origin. To investigate this possibility, we ran a control study and asked participants to report their subjective perception of phosphenes, flickers, and somatosensation. In line with previous literature that showed phosphenes would likely be more prevalent during the frontal montage (DLPFC) closer to the eyes (Schutter \& Hortensius, 2010), our results showed higher ratings of phosphenes and flicker when alpha-tACS was applied to the left DLPFC. We did not find any significant difference in performance in these conditions in our study. Conversely, the significant difference in visual matching performance due to tACS-sensory phase alignment was only with theta-tACS applied to occipital cortex.

Our results could also be interpreted as being due to peripheral tACS effects interfering with readiness to respond as the main difference we observed was in RTs rather than accuracy. If so, accuracy would be higher in the sham group compared with active tACS groups. We did not find any significant differences between the PC when we compared sham $0^{\circ}$ phase condition to $0^{\circ}$ theta occipital and DLPFC groups, or when we compared sham $180^{\circ}$ phase condition to $180^{\circ}$ theta occipital and DLPFC groups. Although the participants were instructed to respond as accurately and as fast as possible, the slow RTs might indicate that they prioritized accuracy over speed. Consequently, it would be likely that the main differences would be observed in the RTs rather than accuracy.
A significant limitation that should be considered is that, in our study, we have focused on the phase synchrony between visual and electrical stimulation rather than the exact timing of the underlying cortical activity. However, there is a lag around 10-60 msec between visual stimulus and visual cortex response, varying among different attributes of the stimulus and individuals (Zeki, 2015; Foxe \& Simpson, 2002; Bair, 1999). It is possible that theta stimulation was less affected by this lag due to its longer period. This could be the reason we did not observe a significant effect in the alpha group. It is, however, interesting for translational and applied neuroscience that stimulation synchrony between tACS and sensory stimulation can have a facilitatory effect even though it may not be expected when taking account of the transmission delay between the eye and the visual cortex. It is possible that, for relatively low frequencies, as used here, the phase difference between the two types of synchronous stimulation due to transmission delays is negligible, and it may be that stronger effects can be observed by accounting for the transmission delay. In the future, measuring the time lag between stimulus and the visual evoked response for each participant before the task using EEG and adjusting the tACS trigger individually can make it possible to address this issue more efficiently and contribute to explaining exactly how the underlying cortical activity modulates perception. However, this has technical issues primarily because tACS induces complex EEG artifacts during stimulation. Very recent work proposes methods for removing these artifacts, but this is subject to verification that the underlying EEG signal is not distorted (Kohli \& Casson, 2019).

Our study contributes to the recently developing literature on concurrent use of tACS and periodic visual stimulation as a promising research technique in neuroscience (Chai et al., 2018; Ruhnau et al., 2016) and the importance of phase synchrony between them (Fiene et al., 2019) and demonstrate a behavioral effect for the first time. This will be of interest to researchers using electrical brain stimulation and sensory stimulation techniques. It might also have implications for applied researchers focusing on the therapeutic and performance enhancement uses of electrical brain stimulation. Furthermore, the spatial, frequency, and phase specificity of our findings would be significant for all researchers interested in the biological and functional organization of perception.

Reprint requests should be sent to Amir-Homayoun Javadi, School of Psychology, Keynes College, University of Kent, CT2 7NP, Canterbury, Kent, United Kingdom, or via e-mail: a.h.javadi@gmail.com.

\section{REFERENCES}

Accornero, N., Capozza, M., Pieroni, L., Pro, S., Davì, L., \& Mecarelli, O. (2014). EEG mean frequency changes in healthy subjects during prefrontal transcranial direct current stimulation. Journal of Neurophysiology, 112, 1367-1375. 
Babiloni, C., Vecchio, F., Bultrini, A., Luca Romani, G., \& Rossini, P. M. (2005). Pre- and poststimulus alpha rhythms are related to conscious visual perception: A high-resolution EEG study. Cerebral Cortex, 16, 1690-1700.

Bair, W. (1999). Spike timing in the mammalian visual system. Current Opinion in Neurobiology, 9, 447-453.

Başar, E., Başar-Eroğlu, C., Karakaş, S., \& Schürmann, M. (2000). Brain oscillations in perception and memory. International Journal of Psychophysiology, 35, 95-124.

Başar, E., Başar-Eroglu, C., Karakaş, S., \& Schürmann, M. (2001). Gamma, alpha, delta, and theta oscillations govern cognitive processes. International Journal of Psychophysiology, 39, 241-248.

Belluscio, M. A., Mizuseki, K., Schmidt, R., Kempter, R., \& Buzsáki, G. (2012). Cross-frequency phase-phase coupling between theta and gamma oscillations in the hippocampus. Journal of Neuroscience, 32, 423-435.

Brooks, J. L., \& Driver, J. (2010). Grouping puts figure-ground assignment in context by constraining propagation of edge assignment. Attention, Perception, \& Psychophysics, 72, 1053-1069.

Brooks, J. L., \& Palmer, S. E. (2011). Cue competition affects temporal dynamics of edge-assignment in human visual cortex. Journal of Cognitive Neuroscience, 23, 631-644.

Bruyer, R., \& Brysbaert, M. (2011). Combining speed and accuracy in cognitive psychology: Is the inverse efficiency score (IES) a better dependent variable than the mean reaction time (RT) and the percentage of errors (PE)? Psychologica Belgica, 51, 5-13.

Busch, N. A., Dubois, J., \& VanRullen, R. (2009). The phase of ongoing EEG oscillations predicts visual perception. Journal of Neuroscience, 29, 7869-7876.

Busch, N. A., \& VanRullen, R. (2010). Spontaneous EEG oscillations reveal periodic sampling of visual attention. Proceedings of the National Academy of Sciences, U.S.A., 107, 16048-16053.

Buzsáki, G., \& Draguhn, A. (2004). Neuronal oscillations in cortical networks. Science, 304, 1926-1929.

Chai, Y., Sheng, J., Bandettini, P. A., \& Gao, J. H. (2018). Frequency-dependent tACS modulation of BOLD signal during rhythmic visual stimulation. Human Brain Mapping, 39, 2111-2120.

Clouter, A., Shapiro, K. L., \& Hanslmayr, S. (2017). Theta phase synchronization is the glue that binds human associative memory. Current Biology, 27, 3143.e6-3148.e6.

Crowley, R., Bendor, D., \& Javadi, A.-H. (2019). A review of neurobiological factors underlying the selective enhancement of memory at encoding, consolidation, and retrieval. Progress in Neurobiology, 179, 101615.

Curtis, C. E., \& D'Esposito, M. (2003). Persistent activity in the prefrontal cortex during working memory. Trends in Cognitive Sciences, 7, 415-423.

Darvas, F., Miller, K. J., Rao, R. P., \& Ojemann, J. G. (2009). Nonlinear phase-phase cross-frequency coupling mediates communication between distant sites in human neocortex. Journal of Neuroscience, 29, 426-435.

Demiralp, T., Bayraktaroglu, Z., Lenz, D., Junge, S., Busch, N. A., Maess, B., et al. (2007). Gamma amplitudes are coupled to theta phase in human EEG during visual perception. International Journal of Psychophysiology, 64, 24-30.

Engel, A. K., Fries, P., \& Singer, W. (2001). Dynamic predictions: Oscillations and synchrony in top-down processing. Nature Reviews Neuroscience, 2, 704-716.

Fertonani, A., \& Miniussi, C. (2017). Transcranial electrical stimulation: What we know and do not know about mechanisms. Neuroscientist, 23, 109-123.

Fiebelkorn, I. C., Saalmann, Y. B., \& Kastner, S. (2013). Rhythmic sampling within and between objects despite sustained attention at a cued location. Current Biology, 23, $2553-2558$

Fiene, M., Schwab, B., Misselhorn, J., Herrmann, C., Schneider, T., \& Engel, A. (2019). Phase-specific aftereffects of transcranial alternating current stimulation on visual processing. Brain Stimulation, 12, 421.

Foxe, J. J., \& Simpson, G. V. (2002). Flow of activation from V1 to frontal cortex in humans. Experimental Brain Research, 142, 139-150.

Händel, B. F., Haarmeier, T., \& Jensen, O. (2011). Alpha oscillations correlate with the successful inhibition of unattended stimuli. Journal of Cognitive Neuroscience, 23, 2494-2502.

Hanslmayr, S., Volberg, G., Wimber, M., Dalal, S. S., \& Greenlee, M. W. (2013). Prestimulus oscillatory phase at $7 \mathrm{~Hz}$ gates cortical information flow and visual perception. Current Biology, 23, 2273-2278.

Helfrich, R. F., Schneider, T. R., Rach, S., Trautmann-Lengsfeld, S. A., Engel, A. K., \& Herrmann, C. S. (2014). Entrainment of brain oscillations by transcranial alternating current stimulation. Current Biology, 24, 333-339.

Henry, M. J., \& Obleser, J. (2012). Frequency modulation entrains slow neural oscillations and optimizes human listening behavior. Proceedings of the National Academy of Sciences, U.S.A., 109, 20095-20100.

Herbst, S. K., Javadi, A.-H., van der Meer, E., \& Busch, N. A. (2013). How long depends on how fast-Perceived flicker dilates subjective duration. PLoS One, 8, e76074.

Herring, J. D., Esterer, S., Marshall, T. R., Jensen, O., \& Bergmann, T. O. (2019). Low- frequency alternating current stimulation rhythmically suppresses gamma-band oscillations and impairs perceptual performance. Neuroimage, 184 , 440-449.

Herrmann, C. S. (2001). Human EEG responses to 1-100 Hz flicker: Resonance phenomena in visual cortex and their potential correlation to cognitive phenomena. Experimental Brain Research, 137, 346-353.

Isler, J. R., Grieve, P. G., Czernochowski, D., Stark, R. I., \& Friedman, D. (2008). Cross-frequency phase coupling of brain rhythms during the orienting response. Brain Research, 1232, 163-172.

Jaegle, A., \& Ro, T. (2014). Direct control of visual perception with phase-specific modulation of posterior parietal cortex. Journal of Cognitive Neuroscience, 26, 422-432.

Javadi, A.-H., Glen, J. C., Halkiopoulos, S., Schulz, M., \& Spiers, H. J. (2017). Oscillatory reinstatement enhances declarative memory. Journal of Neuroscience, 37, 9939-9944.

Javadi, A.-H., \& Walsh, V. (2012). Transcranial direct current stimulation (tDCS) of the left dorsolateral prefrontal cortex modulates declarative memory. Brain Stimulation, 5 , 231-241.

Kanai, R., Chaieb, L., Antal, A., Walsh, V., \& Paulus, W. (2008). Frequency-dependent electrical stimulation of the visual cortex. Current Biology, 18, 1839-1843.

Kienitz, R., Schmiedt, J. T., Shapcott, K. A., Kouroupaki, K., Saunders, R. C., \& Schmid, M. C. (2018). Theta rhythmic neuronal activity and reaction times arising from cortical receptive field interactions during distributed attention. Current Biology, 28, 2377-2387.

Kohli, S., \& Casson, A. (2019). Removal of gross artifacts of transcranial alternating current stimulation in simultaneous EEG monitoring. Sensors, 19, E190.

Lakatos, P., Shah, A. S., Knuth, K. H., Ulbert, I., Karmos, G., \& Schroeder, C. E. (2005). An oscillatory hierarchy controlling neuronal excitability and stimulus processing in the auditory cortex. Journal of Neurophysiology, 94, 1904-1911.

Landau, A. N., \& Fries, P. (2012). Attention samples stimuli rhythmically. Current Biology, 22, 1000-1004. 
Landau, A. N., Schreyer, H. M., Van Pelt, S., \& Fries, P. (2015) Distributed attention is implemented through theta-rhythmic gamma modulation. Current Biology, 25, 2332-2337.

Marg, E., \& Rudiak, D. (1994). Phosphenes induced by magnetic stimulation over the occipital brain: Description and probable site of stimulation. Optometry and Vision Science, 71, 301-311.

Mathewson, K. E., Gratton, G., Fabiani, M., Beck, D. M., \& Ro, T. (2009). To see or not to see: Prestimulus $\alpha$ phase predicts visual awareness. Journal of Neuroscience, 29, 2725-2732.

Neuling, T., Rach, S., Wagner, S., Wolters, C. H., \& Herrmann, C. S. (2012). Good vibrations: Oscillatory phase shapes perception. Neuroimage, 63, 771-778.

Ng, B. S. W., Schroeder, T., \& Kayser, C. (2012). A precluding but not ensuring role of entrained low-frequency oscillations for auditory perception. Journal of Neuroscience, 32, 12268-12276.

Peirce, J. W., Gray, J. R., Simpson, S., MacAskill, M. R., Höchenberger, R., Sogo, H., et al. (2019). PsychoPy2 Experiments in behavior made easy. Behavior Research Methods, 51, 195-203.

Prinzmetal, W., McCool, C., \& Park, S. (2005). Attention: Reaction time and accuracy reveal different mechanisms. Journal of Experimental Psychology: General, 134, 73-92.

Rice, D. M., \& Hagstrom, E. C. (1989). Some evidence in support of a relationship between human auditory signaldetection performance and the phase of the alpha cycle. Perceptual and Motor Skills, 69, 451-457.

Riecke, L., Formisano, E., Herrmann, C. S., \& Sack, A. T. (2015). 4-Hz transcranial alternating current stimulation phase modulates hearing. Brain Stimulation, 8, 777-783.

Ruhnau, P., Keitel, C., Lithari, C., Weisz, N., \& Neuling, T. (2016). Flicker-driven responses in visual cortex change during matched-frequency transcranial alternating current stimulation. Frontiers in Human Neuroscience, 10, 184.

Schaeffner, L. F., \& Welchman, A. E. (2017). Mapping the visual brain areas susceptible to phosphene induction through brain stimulation. Experimental Brain Research, 235, 205-217.

Scheffer-Teixeira, R., \& Tort, A. B. (2016). On cross-frequency phase-phase coupling between theta and gamma oscillations in the hippocampus. elife, 5, e20515.
Schutter, D. J., \& Hortensius, R. (2010). Retinal origin of phosphenes to transcranial alternating current stimulation. Clinical Neurophysiology, 121, 1080-1084.

Spaak, E., Bonnefond, M., Maier, A., Leopold, D. A., \& Jensen, O. (2012). Layer-specific entrainment of gamma-band neural activity by the alpha rhythm in monkey visual cortex. Current Biology, 22, 2313-2318.

Spaak, E., de Lange, F. P., \& Jensen, O. (2014). Local entrainment of alpha oscillations by visual stimuli causes cyclic modulation of perception. Journal of Neuroscience, 34, 3536-3544.

Spyropoulos, G., Bosman, C. A., \& Fries, P. (2018). A theta rhythm in macaque visual cortex and its attentional modulation. Proceedings of the National Academy of Sciences, U.S.A., 115, E5614-E5623.

Thut, G., Nietzel, A., Brandt, S. A., \& Pascual-Leone, A. (2006). A-band electroencephalographic activity over occipital cortex indexes visuospatial attention bias and predicts visual target detection. Journal of Neuroscience, 26, 9494-9502.

Thut, G., Schyns, P., \& Gross, J. (2011). Entrainment of perceptually relevant brain oscillations by non-invasive rhythmic stimulation of the human brain. Frontiers in Psychology, 2, 170.

Tomassini, A., Ambrogioni, L., Medendorp, W. P., \& Maris, E. (2017). Theta oscillations locked to intended actions rhythmically modulate perception. eLife, 6, e25618.

Townsend, J. T., \& Ashby, F. G. (1978). Methods of modeling capacity in simple processing systems. Cognitive Theory, 3 , 200-239.

van Ede, F., de Lange, F. P., \& Maris, E. (2012). Attentional cues affect accuracy and reaction time via different cognitive and neural processes. Journal of Neuroscience, 32, 10408-10412.

Xu, X., Liu, C., Li, Z., \& Zhang, T. (2015). Effects of hydrogen sulfide on modulation of theta-gamma coupling in hippocampus in vascular dementia rats. Brain topography, 28, 879-894

Zeki, S. (2015). A massively asynchronous, parallel brain. Philosophical Transactions of the Royal Society of London, Series B: Biological Sciences, 370, 20140174.

Zheng, C., Bieri, K. W., Hsiao, Y. T., \& Colgin, L. L. (2016) Spatial sequence coding differs during slow and fast gamma rhythms in the hippocampus. Neuron, 89, 398-408. 